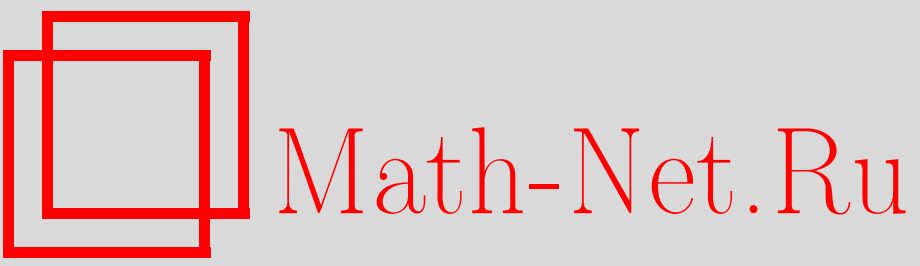

Б. Б. Комраков, Четырехмерные лоренцевы однородные неразрешимые пространства Эйнштейна, УМН, 1997, том 52, выпуск 2, 169-170

DOI: https://doi.org/10.4213/rm835

Использование Общероссийского математического портала Math-Net.Ru подразумевает, что вы прочитали и согласны с пользовательским соглашением

http://www . mathnet.ru/rus/agreement

Параметры загрузки:

IP: 18.209 .158 .208

26 апреля 2023 г., 13:40:43 


\title{
ЧЕТЫРЕХМЕРНЫЕ ЛОРЕНЦЕВЫ ОДНОРОДНЫЕ НЕРАЗРЕШИМЫЕ ПРОСТРАНСТВА ЭЙНШТЕЙНА
}

\author{
Б. Б. КОМРАКОВ
}

Псевдориманово пространство называется пространством Эйнштейна, если метрика $g$ удовлетворяет уравнению Эйнштейна $R-\lambda g=0$, где $R$ - тензор Риччи, $\lambda$ - произвольное вещественное число.

В данной работе представлена локальная классификация четырехмерных лоренцевых (допускающих инвариантную псевдориманову метрику сигнатуры $(3,1))$ однородных неразрешимых пространств Эйнштейна. Локальная классификация всех четырехмерных лоренцевых однородных пространств Эйнштейна насчитывает 44 пространства.

Глобальная классификация в случае римановой метрики может быть найдена в [1]. Известна также полная классификация всех четырехмерных римановых однородных пространств [2], [3]. Полная локальная классификация четырехмерных псевдоримановых однородных пространств произвольной сигнатуры представлена в [4] [5].

Пусть $(\bar{\Gamma}, M)$ - однородное пространство, $G=\bar{\Gamma}_{x}$ - стационарная подгруппа произвольной точки $x \in M$, и $(\overline{\mathfrak{g}}, \mathfrak{g})$ - пара алгебр Ли, соответствующая паре групп Ли $(\bar{\Gamma}, G)$. Заметим, что пара $(\overline{\mathfrak{g}}, \mathfrak{g})$ локально однозначно определяет однородное пространство $(\bar{\Gamma}, M)$. Глобальное строение всех однородных пространств, соответствующих данной паре, приводится в [6].

ОПРеДЕЛЕниЕ. Пару $(\overline{\mathfrak{g}}, \mathfrak{g})$ будем назьвать лоренцевой, если соответствующее однородное пространство $(\bar{\Gamma}, M)$ допускает инвариантную лоренцеву метрику.

Пару $(\overline{\mathfrak{g}}, \mathfrak{g})$ будем называть әйнштейн ов ой, если эта метрика удовлетворяет уравнению Эйнштейна.

Пару $(\overline{\mathfrak{g}}, \mathfrak{g})$ будем называть неразрешимой, если алгебра $\overline{\mathfrak{g}}$ неразрешима.

ЗАМЕЧАНИЕ.

1) Через $\mathfrak{r}_{2}=\langle p, q\rangle$ мы будем обозначать следующую алгебру Ли: $[p, q]=p$.

2) Через $\mathfrak{n}_{3}=\langle h, p, q\rangle$ мы будем обозначать алгебру Ли со следующим ненулевым коммутационным соотношением: $[p, q]=h$.

Теорема 1. Любая неразрешимая симметрическая эйнштейнова лоренцева пара коразмерности 4 әквивалентна одной и только одной из следующих пар:

1) $\overline{\mathfrak{g}}=\mathfrak{s l}(2, \mathbb{R}) \times \mathfrak{s l}(2, \mathbb{R}), \quad \mathfrak{g}=\mathfrak{s o}(2) \times \mathfrak{s o}(1,1)$

2) $\overline{\mathfrak{g}}=\mathfrak{s u}(2) \times \mathfrak{s l}(2, \mathbb{R}), \mathfrak{g}=\mathfrak{s o}(2) \times \mathfrak{s o}(1,1)$

3) $\overline{\mathfrak{g}}=\left(\mathfrak{s o}(2,1)<\mathbb{R}^{3}\right) \times \mathbb{R}, \mathfrak{g}=\mathfrak{s o}(2,1)$

4) $\overline{\mathfrak{g}}=\left(\mathfrak{s o}(3)<\mathbb{R}^{3}\right) \times \mathbb{R}, \mathfrak{g}=\mathfrak{s o}(3)$

5) $\overline{\mathfrak{g}}=\mathfrak{s o}(4,1), \mathfrak{g}=\mathfrak{s o}(3,1)$

6) $\overline{\mathfrak{g}}=\mathfrak{s o}(3,2), \quad \mathfrak{g}=\mathfrak{s o}(3,1)$

7) $\overline{\mathfrak{g}}=\mathfrak{s o}(3,1) \wedge \mathbb{R}^{4}, \mathfrak{g}=\mathfrak{s o}(3,1)$

ТЕОРема 2. Любая неразрешимая несимметрическая эйнштейнова лоренцева пара коразмерности 4 әквивалентна одной и только одной из следующих пар:

1) $\overline{\mathfrak{g}}=\mathfrak{s l}(2, \mathbb{R}) \times \mathfrak{r}_{2}, \mathfrak{g}=\mathfrak{s o}(1,1)$

2) $\overline{\mathfrak{g}}=\mathfrak{s u}(2) \times \mathfrak{r}_{2}, \quad \mathfrak{g}=\mathfrak{s o}(2)$

3) $\overline{\mathfrak{g}}=\mathfrak{s} \mathfrak{l}(2, \mathbb{R}) \times \mathfrak{r}_{2}, \mathfrak{g}=\mathfrak{s o}(2)$

4) $\overline{\mathfrak{g}}=\left\{\left(\begin{array}{ccc}0 & 0 & 0 \\ 0 & x & y \\ 0 & z & -x\end{array}\right) \mid x, y, z \in \mathbb{R}\right\}<\mathfrak{n}_{3}, \mathfrak{g}=\left\langle\left(\begin{array}{ccc}0 & 0 & 0 \\ 0 & 0 & 1 \\ 0 & 0 & 0\end{array}\right)-h, p\right\rangle$

5) $\overline{\mathfrak{g}}=\left\{\left(\begin{array}{ccc}x+t & 0 & 0 \\ 0 & x & y \\ 0 & z & t\end{array}\right) \mid x, y, z, t \in \mathbb{R}\right\} \wedge \mathfrak{n}_{3}, \mathfrak{g}=\left\langle\left(\begin{array}{lll}1 & 0 & 0 \\ 0 & 1 & 0 \\ 0 & 0 & 0\end{array}\right),\left(\begin{array}{lll}0 & 0 & 0 \\ 0 & 0 & 1 \\ 0 & 0 & 0\end{array}\right)-h, p\right\rangle$

6) $\overline{\mathfrak{g}}=\left(\mathfrak{s o}(2,1) \oplus \mathbb{R} \mathrm{id}_{\mathbb{R}^{3}}\right)<\mathbb{R}^{3}, \mathfrak{g}=\mathfrak{s o}(2,1)$

7) $\overline{\mathfrak{g}}=\left(\mathfrak{s o}(3) \oplus \mathbb{R i d}_{\mathbb{R}^{3}}\right) \curlywedge \mathbb{R}^{3}, \quad \mathfrak{g}=\mathfrak{s o}(3)$ 


\section{СПИСОК ЛИТЕРАТУРЫ}

[1] Jensen G. R. // J. Differential Geom. 1969. V. 3. P. 309-349. [2] Берар-Бержери Л. // Четырехмерная риманова геометрия. М.: Мир, 1985. C. 45-60. [3] Ishihara S. // J. Math. Soc. Japan. 1955. V. 7. P. 345-370. [4] Komrakov B., Jnr. Four-dimensional pseudo-Riemannian homogeneous spaces. Classification of complex pairs II // Preprint Univ. Oslo. V. 25 (May 1995). [5] Komrakov B., Jnr. Four-dimensional pseudo-Riemannian homogeneous spaces. Classification of real pairs. // Preprint Univ. Oslo. V. 32 (June 1995). [6] Mostow G. // Ann. of Math. 1950. V. 32 .

Принято редколлегией 18.04.1996 